\section{Analysis of the Li KLL Auger Transition on Freshly Exposed Lithium and Lithium Surface Oxide by AES}

Christopher F. Mallinson, ${ }^{\text {a) }}$ James E. Castle, and John F. Watts

The Surface Analysis Laboratory, Department of Mechanical Engineering Sciences, University of Surrey, Guildford, GU2 7XH, UK

(Received 3 September 2013; accepted 3 October 2013; published 18 November 2013)

Lithium scribed in vacuum and a particle of lithium oxide were analysed by AES and lithium metal exposed to atmosphere for $<1 \mathrm{~min}$ was analysed by XPS. The oxide particle embedded in the host metal was analysed at low and high take off angles. AES spectra acquired demonstrate the change in the KLL structure with increasing oxygen concentration. Survey spectra as well as high resolution narrow spectra were acquired and are presented. The presence of lithium carbonate, together with the oxide, formed in air, was confirmed by XPS as it is not easily resolvable using AES. (C) 2013 American Vacuum Society. [http://dx.doi.org/10.1116/11.20130901]

Keywords: lithium Li; lithium oxide $\mathrm{Li}_{2} \mathrm{O}$; Auger electron spectroscopy AES; $x$-ray photoelectron spectroscopy XPS

\author{
Accession \#: 01274 \\ Technique: AES, XPS \\ Host Material: lithium \\ Instrument: Thermo Scientific Theta \\ Probe, Thermo MicroLab 350 \\ Major Elements in Spectra: Li, O \\ Minor Elements in Spectra: $\mathrm{Na}, \mathrm{C}$, \\ Ar \\ Published Spectra: 11 \\ Spectra in Electronic Record: 24 \\ Spectral Category: comparison
}

\section{INTRODUCTION}

Lithium, the third element of the periodic table, is notable for its widespread use in electrical batteries (Ref. 1), in quartz crystal vibrators, where it improves the Q factor (Ref. 2), in silicon-based x-ray detectors (Ref. 3), and in specialised electronic applications, such as electrochromic thin films (Ref. 4). $\mathrm{LiOH}$ has been used in water treatment for water cooled nuclear power plants (Ref. 5), and, as an alloying element with aluminium alloys (Ref. 6), it is used to gain both weight reduction and improved mechanical properties. Because of its low atomic number it has only a single core level, the $1 \mathrm{~s}$ transition in the metal giving a peak at a binding energy of $54.7 \mathrm{eV}-\mathrm{a}$ value very close to the $3 \mathrm{p}$ level of iron. It is thus easily masked within the spectrum.

In this work spectra are presented that show the main Auger transition for lithium and lithium oxide. The lithium metal differential spectrum shows the primary peak at $53.8 \mathrm{eV}$ together with a loss satellite at $48.8 \mathrm{eV}$. The differential spectrum of the oxide particle, collected with an emission angle of 60 degrees, relative to the sample normal, and a depth of analysis $\sim 0.6$ $\mathrm{nm}$, shows the metal components at 53.8 and $48.5 \mathrm{eV}$ together with two oxide components at 42.5 and $36 \mathrm{eV}$. By tilting the sample 15 degrees from the analyser the take-off angle is reduced and the metal components further suppressed. The differential spectrum of the oxide particle, collected with an emission angle of 75 degrees and a depth of analysis $\sim 0.4 \mathrm{~nm}$, shows a broad feature at $52.7 \mathrm{eV}$, which is likely the remaining metal signal, together with the two main oxide components at 42.5 and $36 \mathrm{eV}$.

A sharp peak at $29 \mathrm{eV}$ in the survey spectrum for scribed lithium metal was been identified as the sodium $\mathrm{L}_{2,3} \mathrm{VV}$ transition, although a narrow scan over the $950-1050 \mathrm{eV}$ region did not show the presence of the sodium KLL transition. The high mobility of sodium in lithium leads to surface segregation with monolayer thickness (Ref. 7).

\footnotetext{
${ }^{a)}$ Author to whom correspondence should be addressed.
}

The kinetic energy of the $\mathrm{Li} \mathrm{KLL}$ and $\mathrm{Li}_{2} \mathrm{O}$ transitions presented here are in good agreement with those previously reported when referencing to the Fermi level is taken into account (Ref. 8).

Gradual oxidation is observed for the bare metal surface over the course of a single analysis even with chamber pressure of $1 \times 10^{-9} \mathrm{mb}$. Re-examining the lithium metal KLL transition at the same sample position after an hour of analysis shows a drop in the intensity of the metal peaks and the appearance of slight oxide peaks. Gradual oxidation can also be observed in the generated SEM image with the formation of islands of oxide.

XPS analysis carried out on a piece of lithium metal exposed to atmosphere for less than 1 min showed the expected significant oxidation of the surface. The surface was argon ion etched for $10 \mathrm{~min}$ to remove carbon contamination and further etching had little effect on the oxide concentration. The lithium $1 \mathrm{~s}$ peak shows a strong oxide component at $57.0 \mathrm{eV}$ and weaker carbonate and metal components at 58.3 and $55.5 \mathrm{eV}$ respectively. Features believed to be surface and bulk plasmons are observed after the Li 1s, O 1s and O KLL peaks, with an energy separation of approximately $10,18.5$, and $25.6 \mathrm{eV}$ from the main peaks. Sodium is not observed in the initial XPS survey spectrum of freshly cut lithium but becomes gradually enriched at the surface during analysis.

\section{SPECIMEN DESCRIPTION (ACCESSION \#01274)}

Host Material: lithium

CAS Registry \#: 7439-93-2

Host Material Characteristics: homogeneous; solid; polycrystalline; conductor; metal

Chemical Name: Li

Source: Sigma-Aldrich

Host Composition: Li $99.9 \%$

Form: $12.7 \mathrm{~mm}$ diameter rod

Lot \#: 265969 Aldrich 
As Received Condition: Lithium rod was stored under argon atmosphere in a glass Winchester.

Analyzed Region: not specified

Ex Situ Preparation/Mounting: Rapid oxidation of the sample occurred after removal from inert atmosphere. A $2 \mathrm{~mm}$ thick sample was cut from the rod using a scalpel. This was mounted in a sample holder and placed into the spectrometer entry lock within $1 \mathrm{~min}$.

In Situ Preparation: The black surface oxide/carbonate was removed in vacuum using the end of a wobble stick, exposing the bare metal surface. Analysis was carried out on the metal and a small oxide particle embedded in the surface.

Pre-Analysis Beam Exposure: One min to atmospheric conditions while the sample was cut and mounted

Charge Control: A shift of $+0.65 \mathrm{eV}$ is applied for the XPS spectra to correct for $\mathrm{C} 1 \mathrm{~s}$ at $285 \mathrm{eV}$

Temp. During Analysis: $300 \mathrm{~K}$

Pressure During Analysis: $<1 \times 10^{-9} \mathrm{~Pa}$

\section{INSTRUMENT DESCRIPTION (INSTRUMENT 1 OF 2) -}

Manufacturer and Model: Thermo Scientific Theta Probe

Analyzer Type: spherical sector

Detector: multi-channel plate detector

Number of Detector Elements: 128

\section{INSTRUMENT PARAMETERS COMMON TO ALL SPECTRA -}

\section{Spectrometer}

Analyzer Mode: constant pass energy

Throughput $\left(T=E^{N}\right)$ : N=See comment below

Throughput Comment: The transmission function is calculated from a cubic polynomial fit to a plot of LOG (Peak Area* $\mathrm{XSF} / \mathrm{PE})$ vs. $\mathrm{LOG}(\mathrm{RR}) \mathrm{RR}=\mathrm{KE} / \mathrm{PE}($ Retard Ratio $=$ Kinetic Energy/Pass Energy) XSF is a term to account for the difference in absolute sensitivities of the Ag MNN and Ag 3d peaks.

Excitation Source Window: none

Excitation Source: $\mathrm{Al} K_{\alpha}$, monochromatic

Source Energy: $1486.68 \mathrm{eV}$

Source Strength: $300 \mathrm{~W}$

Source Beam Size: $200 \mu \mathrm{m} \times 200 \mu \mathrm{m}$

Signal Mode: pulse single channel

\section{Geometry}

Incident Angle: $30^{\circ}$

Source to Analyzer Angle: $67.4^{\circ}$

Emission Angle: $53^{\circ}$

Specimen Azimuthal Angle: $70^{\circ}$

Acceptance Angle from Analyzer Axis: $50 /+30^{\circ}$

Analyzer Angular Acceptance Width: $25^{\circ} \times 25^{\circ}$

\section{Ion Gun}

Manufacturer and Model: Thermo EX05

Energy: $3000 \mathrm{eV}$

Current: $2 \mathrm{~mA}$

Current Measurement Method: biased stage

Sputtering Species: $\mathrm{Ar}^{+}$

Spot Size (unrastered): $200 \mu \mathrm{m}$

Raster Size: $2000 \mu \mathrm{m} \times 2000 \mu \mathrm{m}$

Incident Angle: $45^{\circ}$

Polar Angle: $58.4^{\circ}$

Azimuthal Angle: $100^{\circ}$

Comment: differentially pumped ion gun

INSTRUMENT DESCRIPTION (INSTRUMENT 2 OF 2)

Manufacturer and Model: Thermo Microlab 350

Analyzer Type: spherical sector

Detector: multi-channel parallel detector

Number of Detector Elements: 6

\section{INSTRUMENT PARAMETERS COMMON TO ALL SPECTRA -}

\section{Spectrometer}

Analyzer Mode: constant retard ratio

Throughput $\left(T=E^{N}\right)$ : N=See comment below

Throughput Comment: The transmission function is calculated from a cubic polynomial fit to a plot of LOG (Peak Area* $\mathrm{XSF} / \mathrm{PE})$ vs. LOG (RR)RR=KE/PE (Retard Ratio = Kinetic Energy/Pass Energy) XSF is a term to account for the difference in absolute sensitivities of the Ag MNN and Ag 3d peaks.

Excitation Source Window: none

Excitation Source: electron beam

Source Energy: $10,000 \mathrm{eV}$

Source Strength: $1.2 \mathrm{nA}$

Source Beam Size: $0.1 \mu \mathrm{m} \times 0.1 \mu \mathrm{m}$

Signal Mode: pulse single channel

\section{Geometry}

Incident Angle: $60^{\circ}$

Source to Analyzer Angle: $60^{\circ}$

Emission Angle: varies by spectrum

Specimen Azimuthal Angle: $0^{\circ}$

Acceptance Angle from Analyzer Axis: $50 /+25^{\circ}$

Analyzer Angular Acceptance Width: $30^{\circ} \times 30^{\circ}$

\section{Ion Gun}

Manufacturer and Model: Thermo EX05

Energy: $3000 \mathrm{eV}$

Current: $1 \mathrm{~mA}$ 
Current Measurement Method: biased stage

Sputtering Species: $\mathrm{Ar}^{+}$

Spot Size (unrastered): $200 \mu \mathrm{m}$

Raster Size: $2000 \mu \mathrm{m} \times 2000 \mu \mathrm{m}$

Incident Angle: $67.5^{\circ}$

Polar Angle: $43^{\circ}$

Azimuthal Angle: $228^{\circ}$

Comment: differentially pumped ion gun

\section{DATA ANALYSIS METHOD}

Energy Scale Correction: For the lithium XPS data the binding energy values can be corrected using $\mathrm{C} 1 \mathrm{~s}$ peak at $285.0 \mathrm{eV}$ using a shift of $+0.65 \mathrm{eV}$.

Recommended Energy Scale Shift: For XPS spectra, Accession \#s 1274-10 through $-12,+0.65 \mathrm{eV}$

Peak Shape and Background Method: The peak fitting function used is a Gaussian - Lorentzian product function with a maximum of $30 \%$ Lorentzian mix. The Shirely function was used for background substraction.

Quantitation Method: Peak height has used for peak amplitude and the FWHM of each peak measured using a Shirley background for AES spectra. Peak area used for peak amplitude for XPS spectra. Peaks were fitted using
ThermoScientific Avantage v4.54 software.Sensitivity factors used in Thermo Scientific Avantage software. Peak library is ALWAG from C.D. Wagner, et al., Surf. Interface Anal., 3. $211(1981)$

\section{ACKNOWLEDGMENTS}

The Authors wish to thank the EPRSC and the Engineering Doctorate Programme at the University of Surrey.

\section{REFERENCES}

1. M. S. Whittingham, Science 192, 1126-1127 (1976).

2. A. A. Ballman, R. A. Laudise, and D. W. Rudd, Appl. Phys. Lett. 8, 53-54 (1966).

3. P. E. Gibbons and N. G. Blamires, J. Sci. Instrum. 42, 862-864 (1965).

4. T. Oi, K. Miyauchi, and K. Uehara, J. Appl. Phys. 53, 18231825 (1982).

5. W. H. Hocking and D. H. Lister, Surf. Interface Anal. 11, 45-59 (1988).

6. J. W. Martin, Annu. Rev. Mater. Sci. 18, 101-119 (1988).

7. G. L. Powell, R. E. Clausing, and G. E. McGuire, Surf. Sci. 49, 310-314 (1975).

8. G. L. Powell, R. E. Clausing, and G. E. McGuire, Surf. Sci. 36, 377-379 (1973). 


\begin{tabular}{|c|c|c|c|c|c|c|c|}
\hline \multicolumn{8}{|c|}{ SPECTRAL FEATURES TABLE } \\
\hline $\begin{array}{l}\text { Spectrum } \\
\text { ID \# }\end{array}$ & $\begin{array}{l}\text { Element/ } \\
\text { Transition }\end{array}$ & $\begin{array}{c}\text { Peak } \\
\text { Energy } \\
(\mathrm{eV})\end{array}$ & $\begin{array}{c}\text { Peak Width } \\
\text { FWHM } \\
(\mathrm{eV})\end{array}$ & $\begin{array}{l}\text { Peak Area } \\
(\mathrm{eV} \times \mathrm{cts} / \mathrm{s})\end{array}$ & $\begin{array}{l}\text { Sensitivity } \\
\text { Factor }\end{array}$ & $\begin{array}{c}\text { Concentration } \\
\text { (at. \%) }\end{array}$ & $\begin{array}{c}\text { Peak } \\
\text { Assignment }\end{array}$ \\
\hline $01274-02^{a}$ & Li KLL & $52.3,46.5: 53$ & 6.8 & 15274 & $\cdots$ & $\cdots$ & Li metal \\
\hline $01274-09^{b}$ & Ox KLL & 509.5 & 6.2 & 626 & $\cdots$ & $\cdots$ & $\mathrm{O}$ in $\mathrm{Li}_{2} \mathrm{O} / \mathrm{LiOH}$ \\
\hline $01274-04^{c}$ & Li KLL & $51.8,40.8: 53$ & 8.9 & 49274 & $\cdots$ & $\cdots$ & $\mathrm{Li}$ in $\mathrm{Li}_{2} \mathrm{O}$ and metal \\
\hline $01274-05^{b}$ & Ox KLL & 509.8 & 5.6 & 89707 & $\cdots$ & $\cdots$ & $\mathrm{O}$ in $\mathrm{Li}_{2} \mathrm{O} / \mathrm{LiOH}$ \\
\hline $01274-07^{d}$ & Li KLL & $40.7,34.3: 42$ & 3.0 & 16603 & $\ldots$ & $\cdots$ & $\mathrm{Li}$ in $\mathrm{Li}_{2} \mathrm{O}$ \\
\hline $01274-08^{b}$ & Ox KLL & 509.8 & 5.0 & 27809 & $\cdots$ & $\cdots$ & $\mathrm{O}$ in $\mathrm{Li}_{2} \mathrm{O} / \mathrm{LiOH}$ \\
\hline $01274-06^{e}$ & Ca KLL & 264.0 & - & 1392 & $\cdots$ & $\cdots$ & Contamination or carbonate \\
\hline 01274-11 & Li 1s & 55.5 & 0.78 & 154 & 0.02 & 2.59 & Li metal \\
\hline 01274-11 & Li 1s & 57.0 & 1.37 & 3295 & 0.02 & 55.5 & $\mathrm{Li}$ in $\mathrm{Li}_{2} \mathrm{O} / \mathrm{LiOH}$ \\
\hline $01274-11^{f}$ & Li 1s & 58.3 & 1.37 & 116 & 0.02 & 1.96 & $\mathrm{Li}$ in $\mathrm{Li}_{2} \mathrm{CO}_{3}$ \\
\hline $01274-12^{f}$ & $01 \mathrm{~s}$ & 534.7 & 1.43 & 3310 & 0.66 & 4.15 & $\mathrm{O}$ in $\mathrm{Li}_{2} \mathrm{CO}_{3}$ \\
\hline 01274-12 & $01 \mathrm{~s}$ & 531.9 & 1.16 & 27922 & 0.66 & 34.97 & $\mathrm{O}$ in $\mathrm{Li}_{2} \mathrm{O} / \mathrm{LiOH}$ \\
\hline $01274-13^{g}$ & $\operatorname{Ar} 2 p$ & 245.8 & 1.27 & 90.8 & 0.96 & 0.03 & $\operatorname{Ar}$ \\
\hline $01274-14^{\mathrm{h}}$ & C 1s & 285.0 & 1.72 & 181 & 0.25 & 0.27 & Hydrocarbon contamination \\
\hline $01274-14^{f}$ & C 1s & 288.8 & 2.41 & 343 & 0.25 & 0.52 & $\mathrm{C}$ in $\mathrm{Li}_{2} \mathrm{CO}_{3}$ \\
\hline
\end{tabular}

a Scribed in vacuum sample

${ }^{b}$ Oxygen could be present at surface in the form of lithium oxide or hydroxide.

${ }^{\circ}$ Oxide particle sample

d Tilted oxide particle sample

${ }^{\mathrm{e}}$ Carbon may be present as trace surface contamination or as lithium carbonate.

${ }^{f}$ Lithium carbonate is formed when lithium reacts with carbon dioxide in the atmosphere.

${ }^{9}$ Argon is gradually implanted into the lithium surface during argon ion etching.

${ }^{\mathrm{h}}$ Contamination from the atmosphere or the scalpel blade used to cut the lithium sample

\begin{tabular}{|c|c|c|c|c|c|c|c|}
\hline \multicolumn{8}{|c|}{ ANALYZER CALIBRATION TABLE } \\
\hline $\begin{array}{l}\text { Spectrum } \\
\text { ID \# }\end{array}$ & $\begin{array}{l}\text { Element/ } \\
\text { Transition }\end{array}$ & $\begin{array}{c}\text { Peak Energy } \\
(\mathrm{eV})\end{array}$ & $\begin{array}{l}\text { Peak Width } \\
\text { FWHM (eV) }\end{array}$ & $\begin{array}{l}\text { Peak Area } \\
(\mathrm{eV} \times \mathrm{cts} / \mathrm{s})\end{array}$ & $\begin{array}{l}\text { Sensitivity } \\
\text { Factor }\end{array}$ & $\begin{array}{c}\text { Concentration } \\
\text { (at. \%) }\end{array}$ & $\begin{array}{c}\text { Peak } \\
\text { Assignment }\end{array}$ \\
\hline 17 & Au $\mathrm{M}_{5} \mathrm{~N}_{6,7} \mathrm{~N}_{6,7}$ & 2014.0 & 22 & 19676 & $\cdots$ & $\cdots$ & Au metal \\
\hline 16 & $\mathrm{Au} \mathrm{N}_{6,7} \mathrm{VV}$ & $69.5,71.5$ & 7.4 & 38605 & $\cdots$ & $\cdots$ & Au metal \\
\hline 22 & $\mathrm{Ag} \mathrm{M}_{4} \mathrm{NN}$ & $351.8,357.5$ & 4.4 & 111196 & $\cdots$ & $\cdots$ & Ag metal \\
\hline 20 & $\mathrm{Cu} \mathrm{L}_{3} \mathrm{VV}$ & $60.2,62.3$ & 6.2 & 19337 & $\cdots$ & $\cdots$ & Cu metal \\
\hline 19 & $\mathrm{Cu} \mathrm{M}_{2,3} \mathrm{VV}$ & 917.3 & 7.2 & 41899 & $\cdots$ & $\cdots$ & Cu metal \\
\hline 23 & $\mathrm{Au} 4 \mathrm{f}_{7 / 2}$ & 84.1 & 0.74 & 17347 & 4.95 & 100 & Au metal \\
\hline 24 & $\mathrm{Cu} 2 \mathrm{p}_{3 / 2}$ & 932.6 & 0.87 & 108427 & 4.2 & 100 & Cu metal \\
\hline
\end{tabular}




\begin{tabular}{|c|c|c|c|c|c|}
\hline \multicolumn{6}{|c|}{ GUIDE TO FIGURES } \\
\hline Spectrum (Accession) \# & Spectral Region & Voltage Shift ${ }^{*}$ & Multiplier & Baseline & Comment \# \\
\hline $1274-01$ & survey & & 1 & 0 & 1 \\
\hline $1274-02$ & Li KLL & & 1 & 0 & 1 \\
\hline $1274-03$ & survey & & 1 & 0 & 2 \\
\hline $1274-04$ & Li KLL & & 1 & 0 & 2 \\
\hline 1274-05 & O KLL & & 1 & 0 & 2 \\
\hline $1274-06$ & survey & & 1 & 0 & 3 \\
\hline $1274-07$ & Li KLL & & 1 & 0 & 3 \\
\hline $1274-08$ & O KLL & & 1 & 0 & 3 \\
\hline $1274-09[N P]^{* *}$ & O KLL & & 1 & 0 & 1 \\
\hline $1274-10$ & survey & -0.65 & 1 & 0 & 4 \\
\hline $1274-11$ & Li 1s & -0.65 & 1 & 0 & 4 \\
\hline $1274-12$ & O 1s & -0.65 & 1 & 0 & 4 \\
\hline 1274-13 [NP] & $\operatorname{Ar} 2 p$ & -0.65 & 1 & 0 & 4 \\
\hline 1274-14 [NP] & C 1s & -0.65 & 1 & 0 & 4 \\
\hline 1275-01 [NP] & Au NVV, Au MNN & & 1 & 0 & 5 \\
\hline 1275-02 [NP] & Au NVV & & 1 & 0 & 5 \\
\hline 1275-03 [NP] & Au MNN & & 1 & 0 & 5 \\
\hline 1275-04 [NP] & Au $4 f$ & & 1 & 0 & 5 \\
\hline 1276-01 [NP] & Cu MVV, Cu LVV & & 1 & 0 & 6 \\
\hline 1276-02 [NP] & Cu MVV & & 1 & 0 & 6 \\
\hline 1276-03 [NP] & Cu LVV & & 1 & 0 & 6 \\
\hline 1276-04 [NP] & Cu 2p & & 1 & 0 & 6 \\
\hline $1277-01$ [NP] & Ag MNN & & 1 & 0 & 7 \\
\hline $1277-02$ [NP] & Ag MNN & & 1 & 0 & 7 \\
\hline
\end{tabular}

*Voltage shift of the archived (as-measured) spectrum relative to the printed figure. The figure reflects the recommended energy scale correction due to a calibration correction, sample charging, flood gun, or other phenomenon.

" [NP] signifies not published; digital spectra are archived in SSS database but not reproduced in the printed journal.

1. fresh Li metal surface, scribed in situ, take-off angle $=30^{\circ}$

2. oxide particle embedded in Li sample, take-off angle $=30^{\circ}$

3. oxide particle embedded in Li sample, take-off angle $=15^{\circ}$

4. surface of Li sample exposed to air for $<1 \mathrm{~min}$, take-off angle $=30^{\circ}$

5. Au calibration

6. Cu calibration

7. Ag calibration 


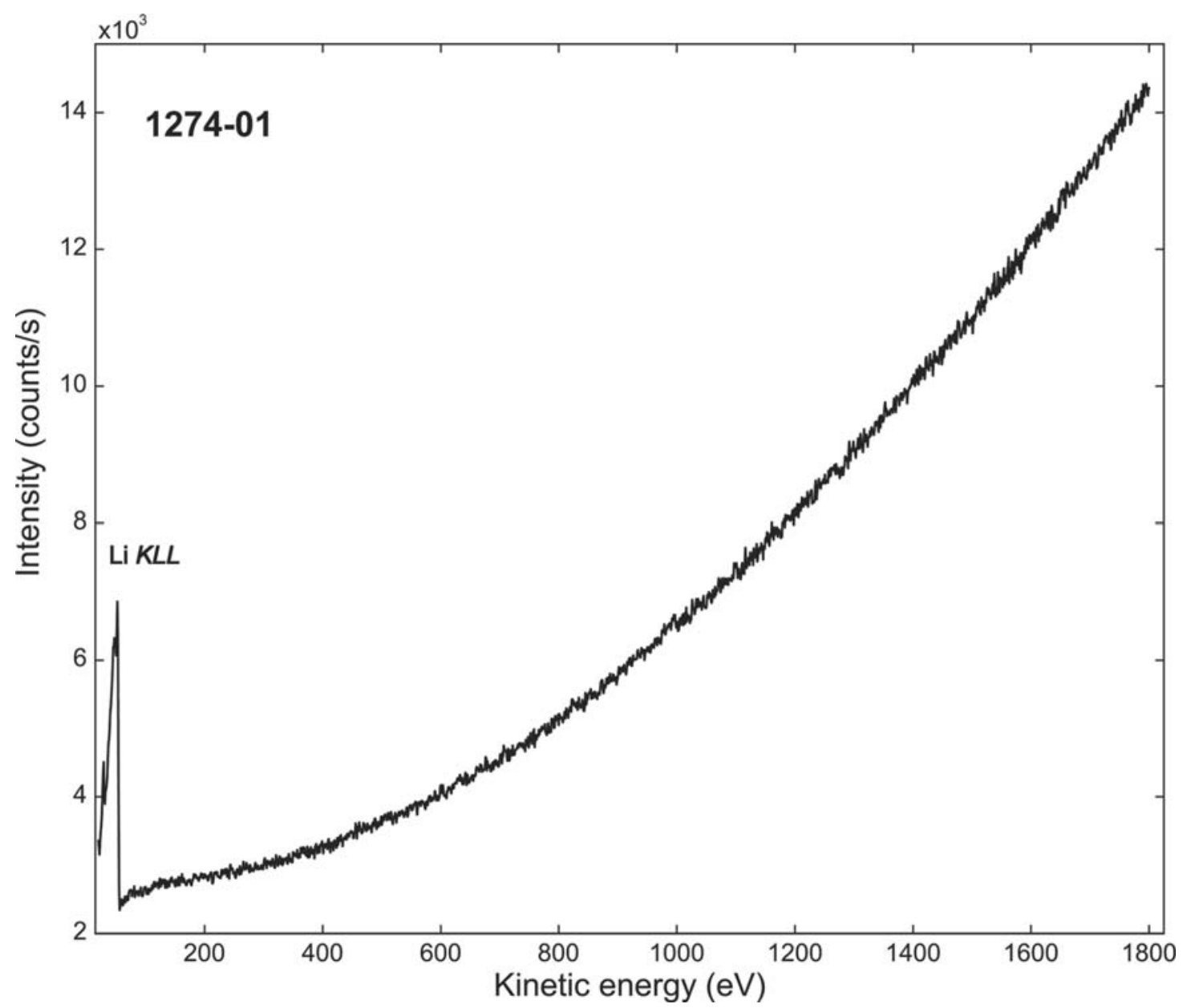

\begin{tabular}{|c|c|}
\hline Accession \# & 01274-01 \\
\hline Host Material & lithium \\
\hline Technique & AES \\
\hline Spectral Region & survey \\
\hline Instrument & Thermo MicroLab 350 \\
\hline Excitation Source & electron beam \\
\hline Source Energy & 10000 eV \\
\hline Source Strength & $1.2 \mathrm{nA}$ \\
\hline Source Size & $0.1 \mu \mathrm{m} \times 0.1 \mu \mathrm{m}$ \\
\hline Analyzer Type & spherical sector \\
\hline Incident Angle & $30^{\circ}$ \\
\hline Emission Angle & $60^{\circ}$ \\
\hline Analyzer Retard Ratio: & 4 \\
\hline Analyzer Resolution & $2 \%$ \\
\hline Total Signal Accumulation Time & $267 s$ \\
\hline Total Elapsed Time & $361 \mathrm{~s}$ \\
\hline Number of Scans & 3 \\
\hline Source Beam Size at Specimen Surface & $0.011 \mu \mathrm{m} \times 0.011 \mu \mathrm{m}$ \\
\hline Effective Detector Width & $1 \mathrm{eV}$ \\
\hline Analyzer Angular Acceptance Width & $25^{\circ} \times 25^{\circ}$ \\
\hline Comment & fresh Li metal surface, scribed in situ, take-off angle $=30^{\circ}$ \\
\hline
\end{tabular}




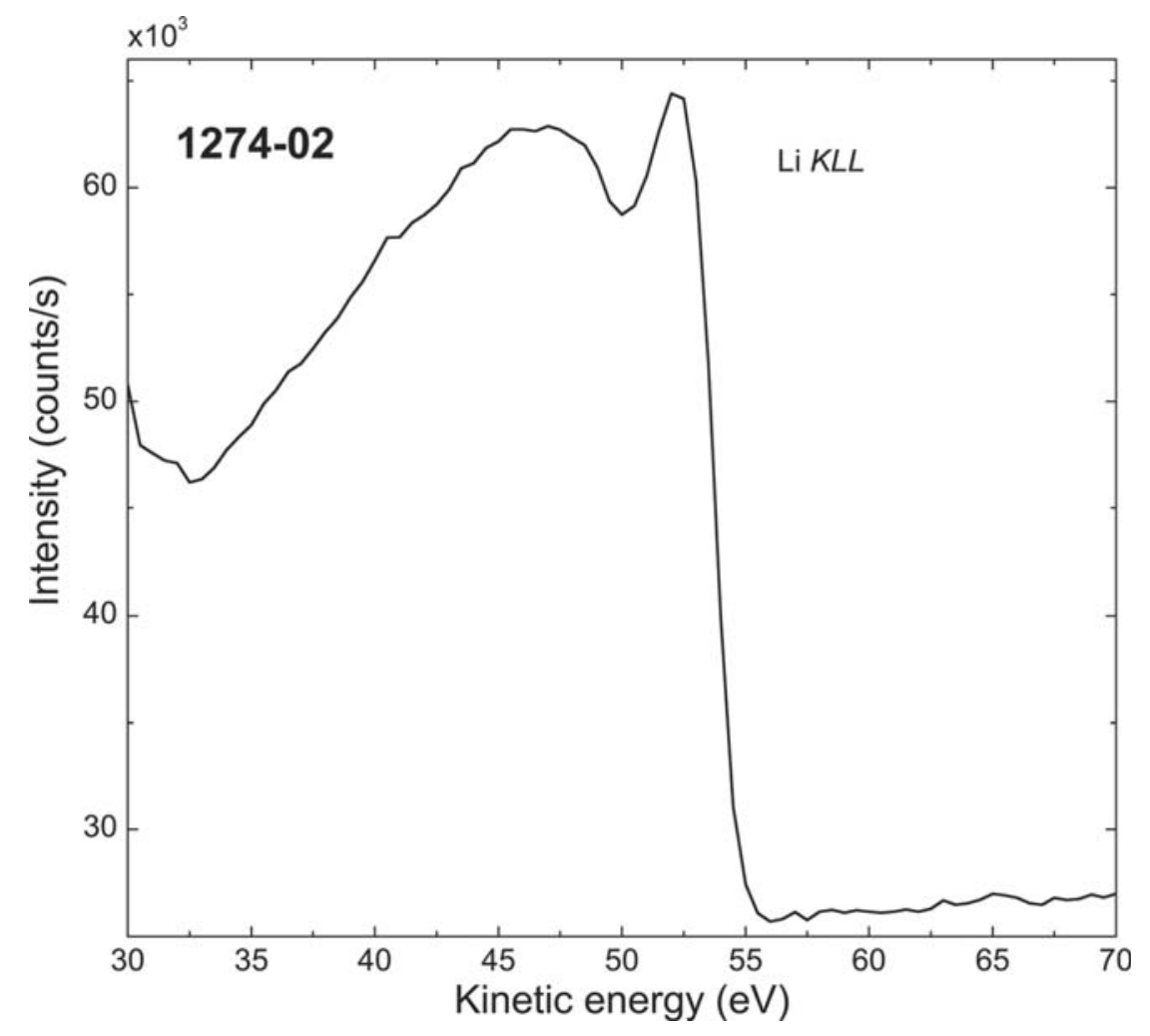

- Accession \#: 01274-02

- Host Material: lithium

- Technique: AES

- Spectral Region: Li KLL

Instrument: Thermo MicroLab 350

Excitation Source: electron beam

Source Energy: $10000 \mathrm{eV}$

Source Strength: $1.2 \mathrm{nA}$

Source Size: $0.1 \mu \mathrm{m} \times 0.1 \mu \mathrm{m}$

Analyzer Type: spherical sector

Incident Angle: $30^{\circ}$

Emission Angle: $60^{\circ}$

Analyzer Retard Ratio: 4

Analyzer Resolution: $2 \%$

Total Signal Accumulation Time: $203 \mathrm{~s}$

Total Elapsed Time: $272 \mathrm{~s}$

Number of Scans: 50

Source Beam Size at Specimen Surface: $0.011 \mu \mathrm{m} \times 0.011 \mu \mathrm{m}$

Effective Detector Width: $0.5 \mathrm{eV}$

Analyzer Angular Acceptance Width: $25^{\circ} \times 25^{\circ}$

Comment: fresh Li metal surface, scribed in situ, take-off angle $30^{\circ}$

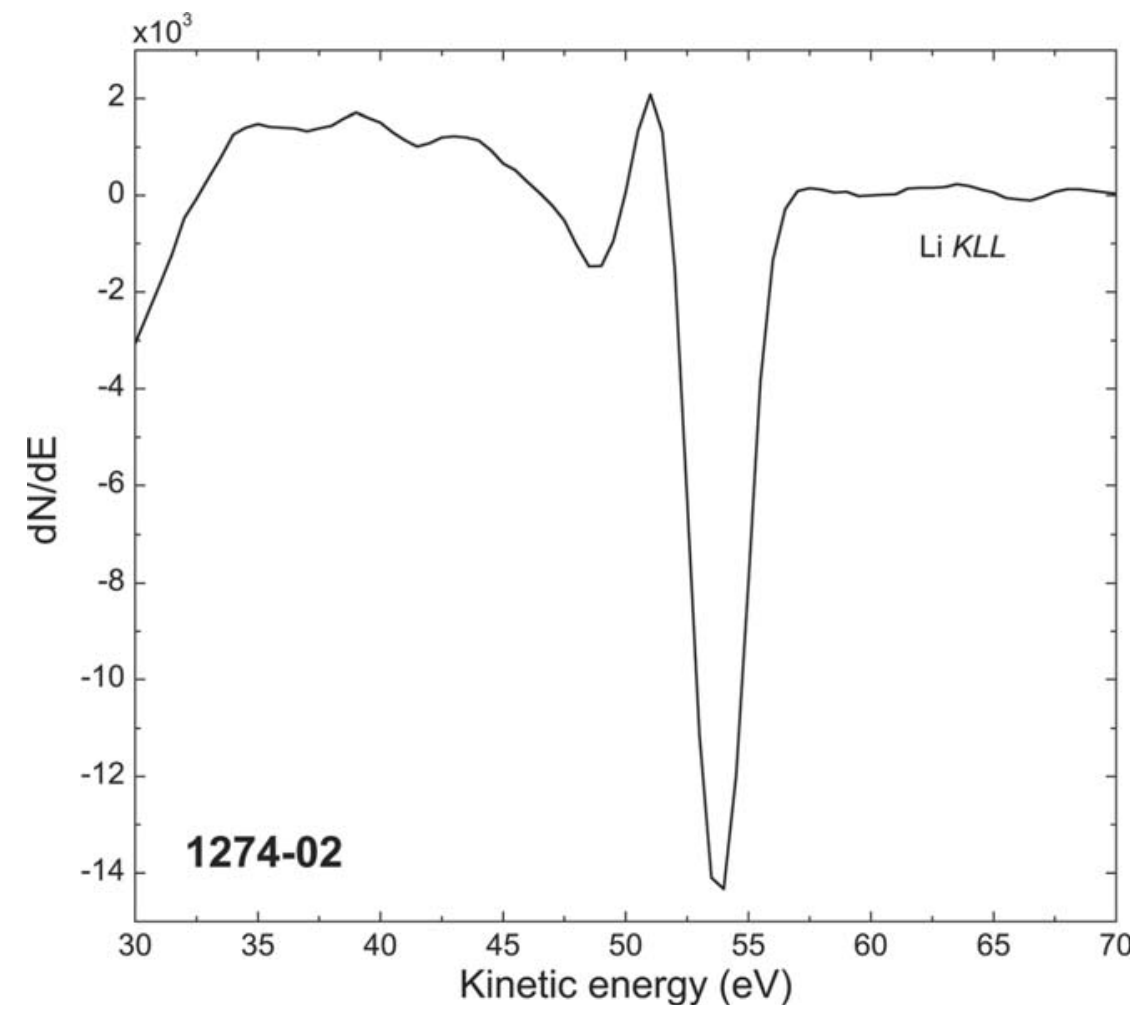




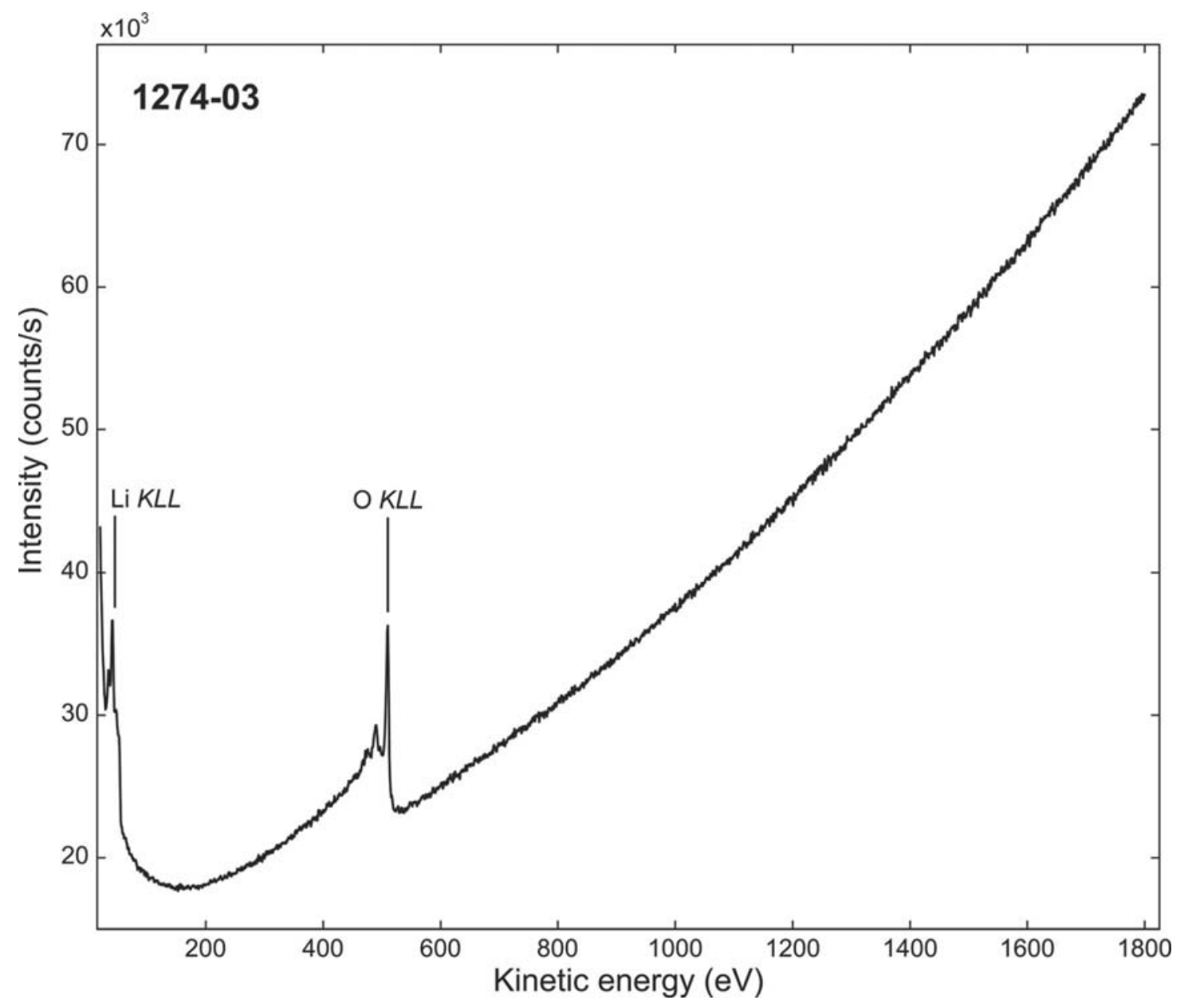

\begin{tabular}{|c|c|}
\hline Accession \# & $01274-03$ \\
\hline Host Material & lithium \\
\hline Technique & AES \\
\hline Spectral Region & survey \\
\hline Instrument & Thermo MicroLab 350 \\
\hline Excitation Source & electron beam \\
\hline Source Energy & $10000 \mathrm{eV}$ \\
\hline Source Strength & $1.2 \mathrm{nA}$ \\
\hline Source Size & $0.1 \mu \mathrm{m} \times 0.1 \mu \mathrm{m}$ \\
\hline Analyzer Type & spherical sector \\
\hline Incident Angle & $30^{\circ}$ \\
\hline Emission Angle & $60^{\circ}$ \\
\hline Analyzer Retard Ratio: & 4 \\
\hline Analyzer Resolution & $2 \%$ \\
\hline Total Signal Accumulation Time & $267 s$ \\
\hline Total Elapsed Time & $361 \mathrm{~s}$ \\
\hline Number of Scans & 3 \\
\hline Source Beam Size at Specimen Surface & $0.011 \mu \mathrm{m} \times 0.011 \mu \mathrm{m}$ \\
\hline Effective Detector Width & $1 \mathrm{eV}$ \\
\hline Analyzer Angular Acceptance Width & $25^{\circ} \times 25^{\circ}$ \\
\hline Comment & oxide particle embedded in Li sample, take-off angle $=30^{\circ}$ \\
\hline
\end{tabular}




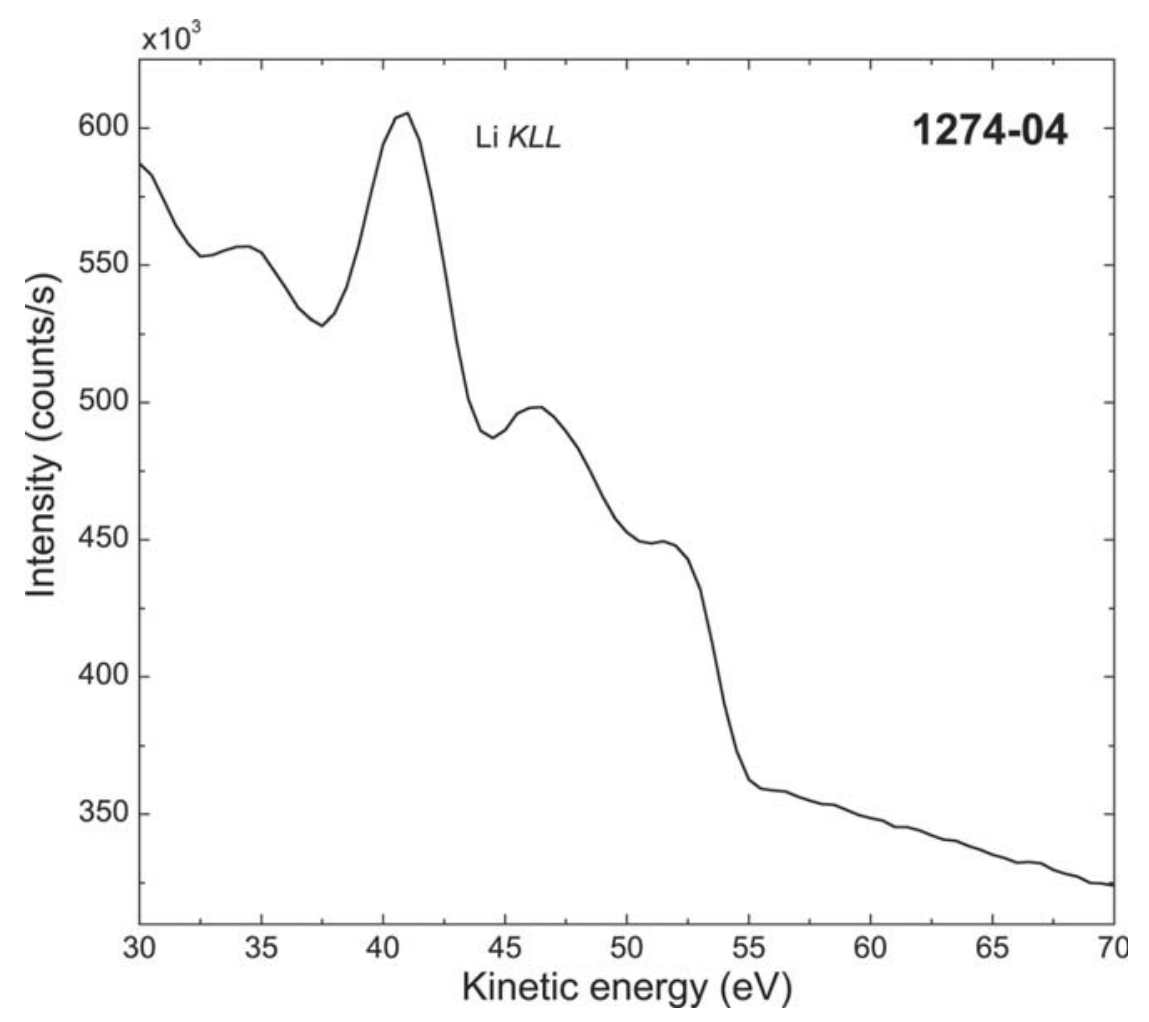

- Accession \#: 01274-04

- Host Material: lithium

- Technique: AES

- Spectral Region: Li KLL

Instrument: Thermo MicroLab 350

Excitation Source: electron beam

Source Energy: $10000 \mathrm{eV}$

Source Strength: $1.2 \mathrm{nA}$

Source Size: $0.1 \mu \mathrm{m} \times 0.1 \mu \mathrm{m}$

Analyzer Type: spherical sector

Incident Angle: $30^{\circ}$

Emission Angle: $60^{\circ}$

Analyzer Retard Ratio: 4

Analyzer Resolution: $2 \%$

Total Signal Accumulation Time: $203 \mathrm{~s}$

Total Elapsed Time: $272 \mathrm{~s}$

Number of Scans: 50

Source Beam Size at Specimen Surface: $0.011 \mu \mathrm{m} \times 0.011 \mu \mathrm{m}$

Effective Detector Width: $0.5 \mathrm{eV}$

Analyzer Angular Acceptance Width: $25^{\circ} \times 25^{\circ}$

Comment: oxide particle embedded in Li sample, take-off angle $=30^{\circ}$

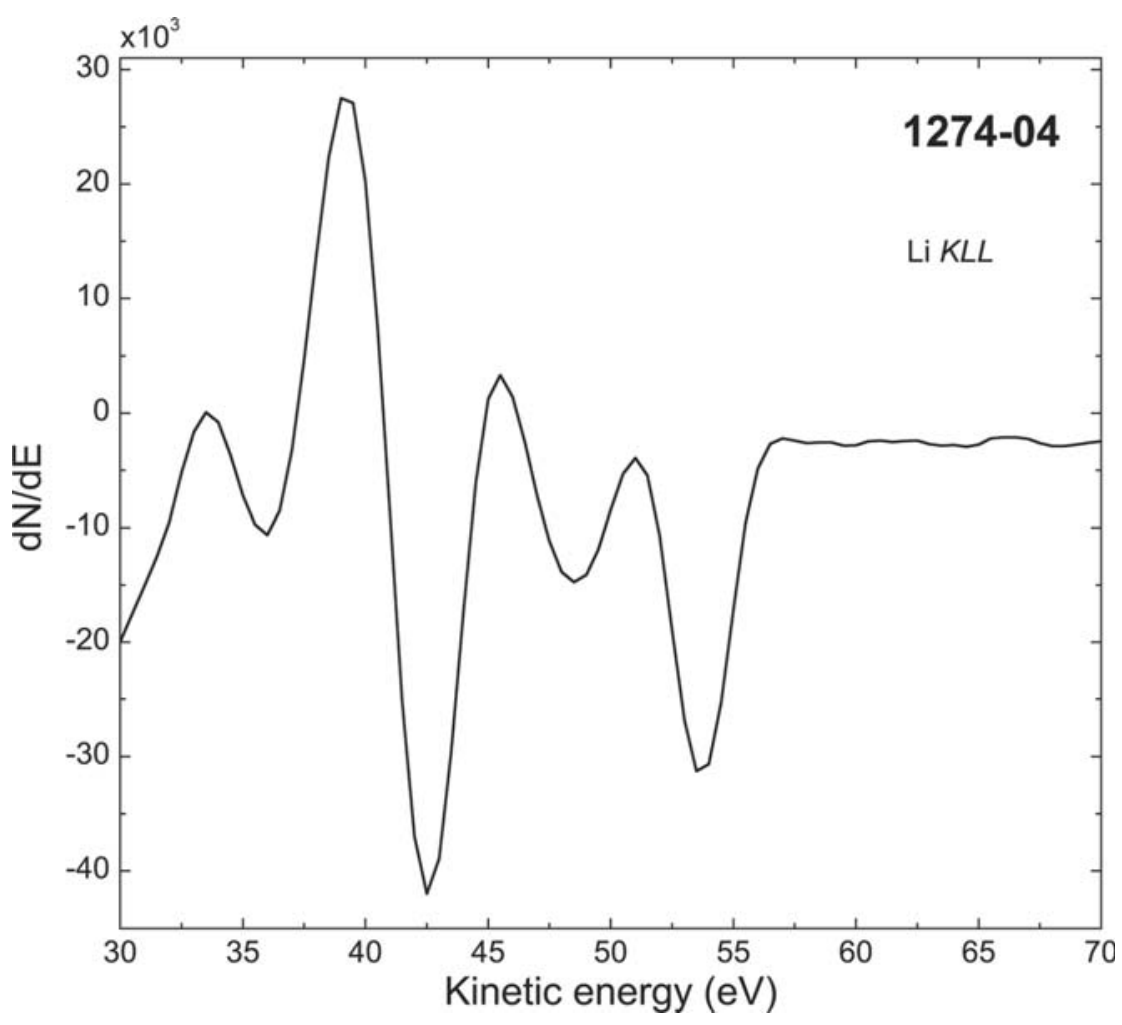




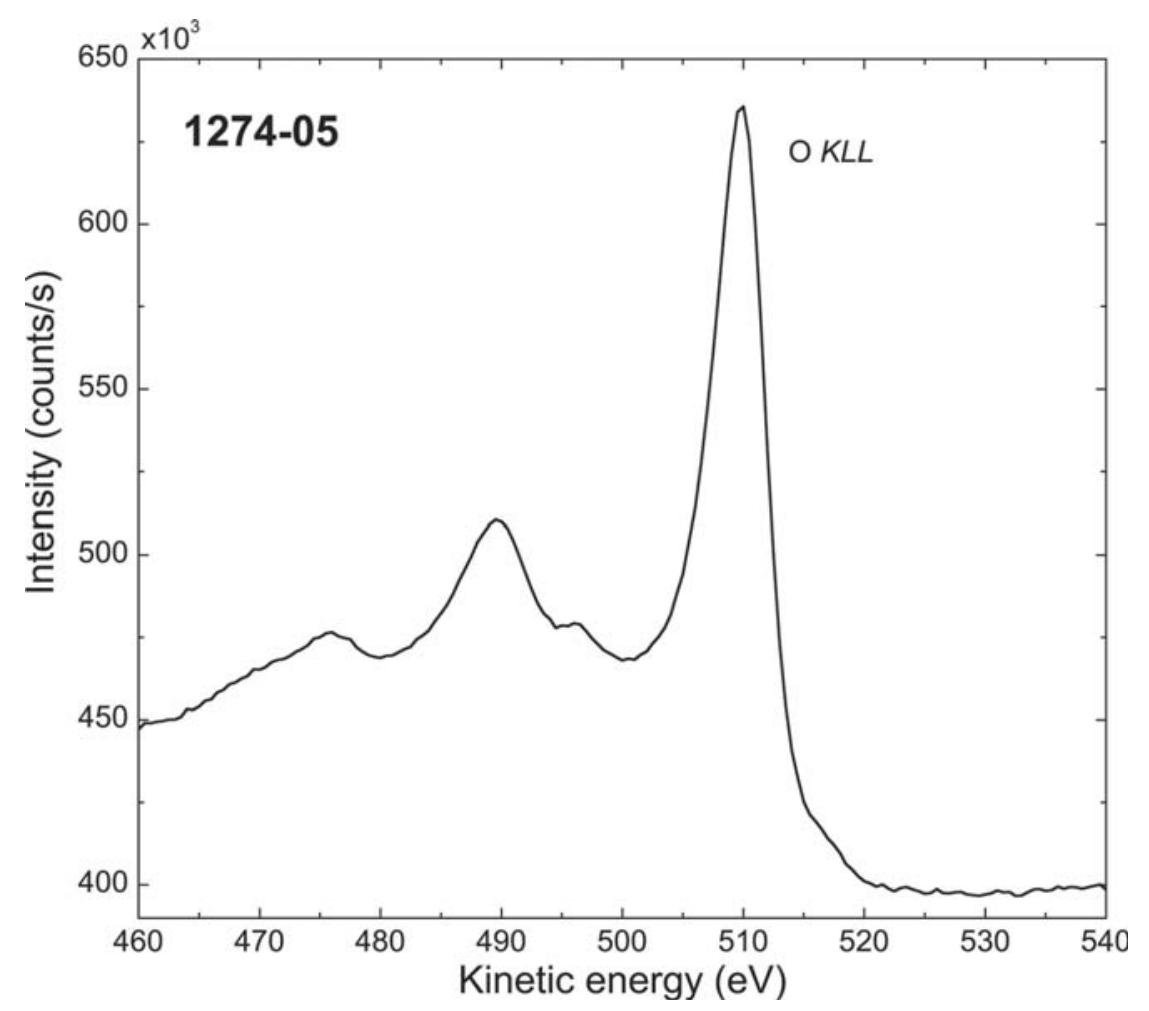

- Accession \#: 01274-05

nost Material: lithium

- Technique: AES

- Spectral Region: O KLL

Instrument: Thermo MicroLab 350

Excitation Source: electron beam

Source Energy: $10000 \mathrm{eV}$

Source Strength: $1.2 \mathrm{nA}$

Source Size: $0.1 \mu \mathrm{m} \times 0.1 \mu \mathrm{m}$

Analyzer Type: spherical sector

Incident Angle: $30^{\circ}$

Emission Angle: $60^{\circ}$

Analyzer Retard Ratio: 4

Analyzer Resolution: 2\%

Total Signal Accumulation Time: $402 \mathrm{~s}$

Total Elapsed Time: $510 \mathrm{~s}$

Number of Scans: 50

Source Beam Size at Specimen Surface: $0.011 \mu \mathrm{m} \times 0.011 \mu \mathrm{m}$

Effective Detector Width: $0.5 \mathrm{eV}$

Analyzer Angular Acceptance Width: $25^{\circ} \times 25^{\circ}$

Comment: oxide particle embedded in Li sample, take-off angle $=30^{\circ}$ 


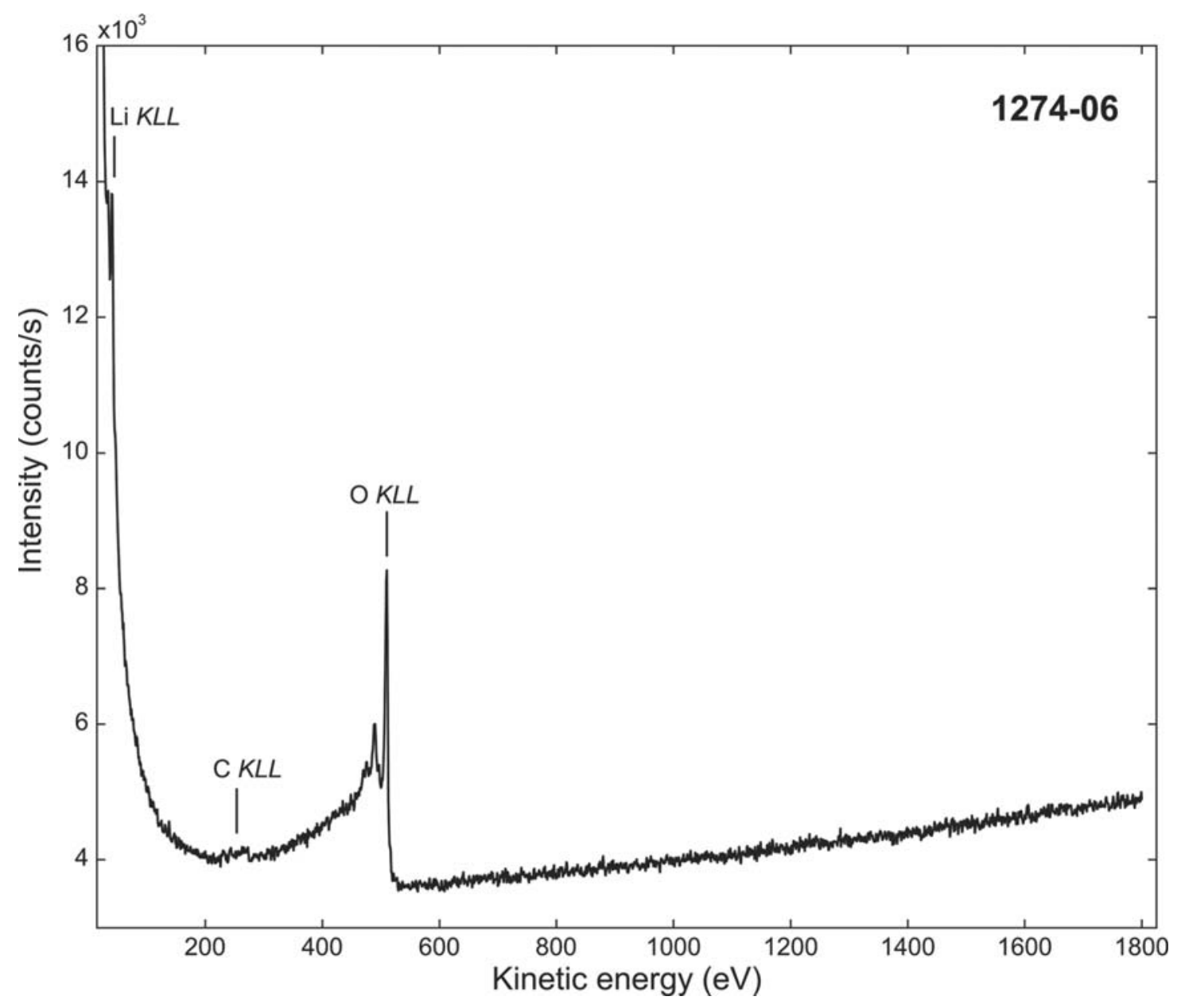

\begin{tabular}{|c|c|}
\hline Accession \# & $01274-06$ \\
\hline Host Material & lithium \\
\hline Technique & AES \\
\hline Spectral Region & survey \\
\hline Instrument & Thermo MicroLab 350 \\
\hline Excitation Source & electron beam \\
\hline Source Energy & $10000 \mathrm{eV}$ \\
\hline Source Strength & $1.2 \mathrm{nA}$ \\
\hline Source Size & $0.1 \mu \mathrm{m} \times 0.1 \mu \mathrm{m}$ \\
\hline Analyzer Type & spherical sector \\
\hline Incident Angle & $30^{\circ}$ \\
\hline Emission Angle & $75^{\circ}$ \\
\hline Analyzer Retard Ratio: & 4 \\
\hline Analyzer Resolution & $2 \%$ \\
\hline Total Signal Accumulation Time & $267 s$ \\
\hline Total Elapsed Time & $361 \mathrm{~s}$ \\
\hline Number of Scans & 3 \\
\hline Source Beam Size at Specimen Surface & $0.011 \mu \mathrm{m} \times 0.011 \mu \mathrm{m}$ \\
\hline Effective Detector Width & $1 \mathrm{eV}$ \\
\hline Analyzer Angular Acceptance Width & $25^{\circ} \times 25^{\circ}$ \\
\hline Comment & oxide particle embedded in Li sample, take-off angle $=15^{\circ}$ \\
\hline
\end{tabular}




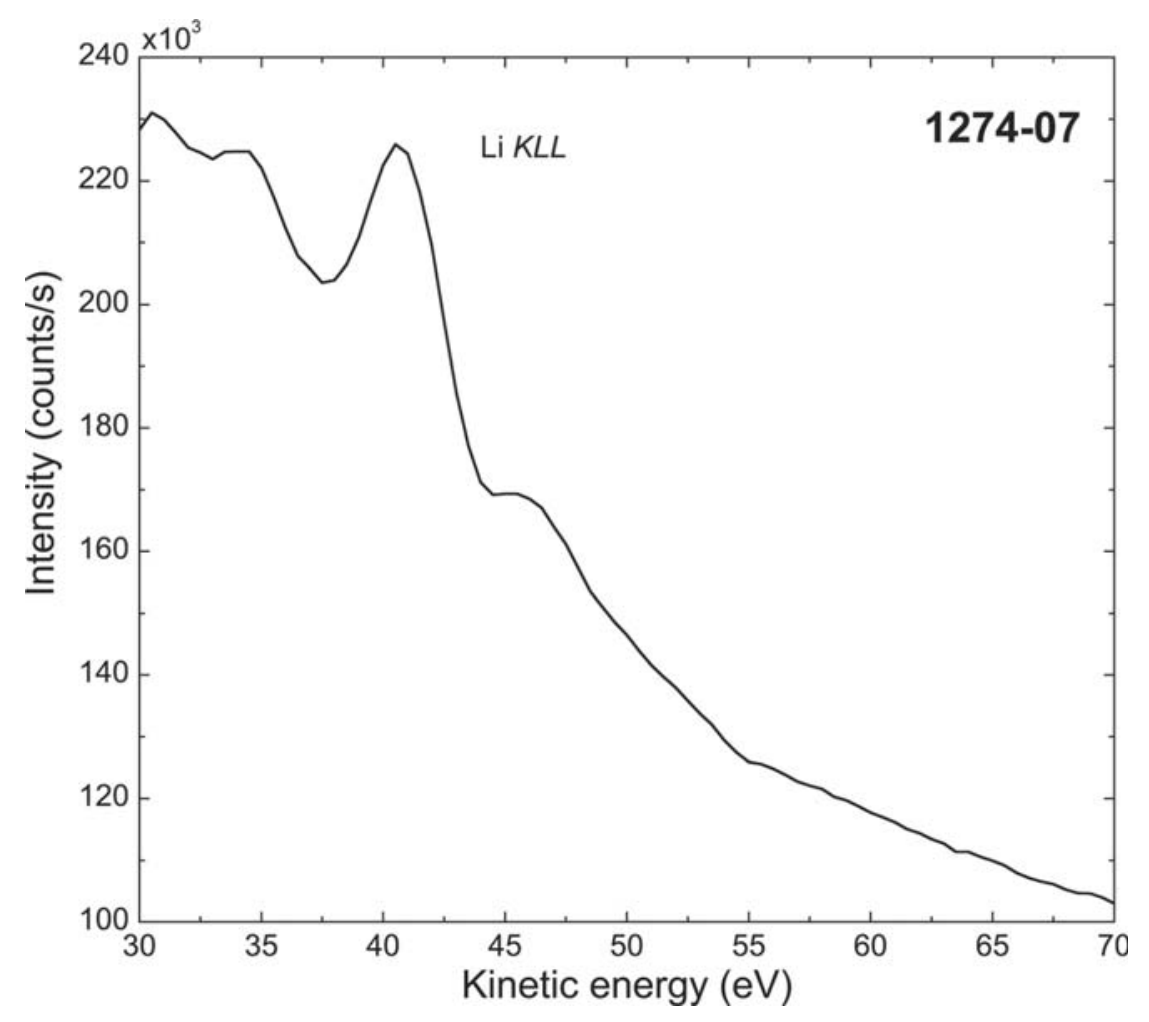

- Accession \#: 01274-07

nost Material: lithium

- Technique: AES

- Spectral Region: Li KLL

Instrument: Thermo MicroLab 350

Excitation Source: electron beam

Source Energy: $10000 \mathrm{eV}$

Source Strength: $1.2 \mathrm{nA}$

Source Size: $0.1 \mu \mathrm{m} \times 0.1 \mu \mathrm{m}$

Analyzer Type: spherical sector

Incident Angle: $30^{\circ}$

Emission Angle: $75^{\circ}$

Analyzer Retard Ratio: 4

Analyzer Resolution: 2\%

Total Signal Accumulation Time: 203 s

Total Elapsed Time: 272 s

Number of Scans: 50

Source Beam Size at Specimen Surface: $0.011 \mu \mathrm{m} \times 0.011 \mu \mathrm{m}$

Effective Detector Width: $0.5 \mathrm{eV}$

Analyzer Angular Acceptance Width: $25^{\circ} \times 25^{\circ}$

Comment: oxide particle embedded in Li sample, take-off angle $=15^{\circ}$

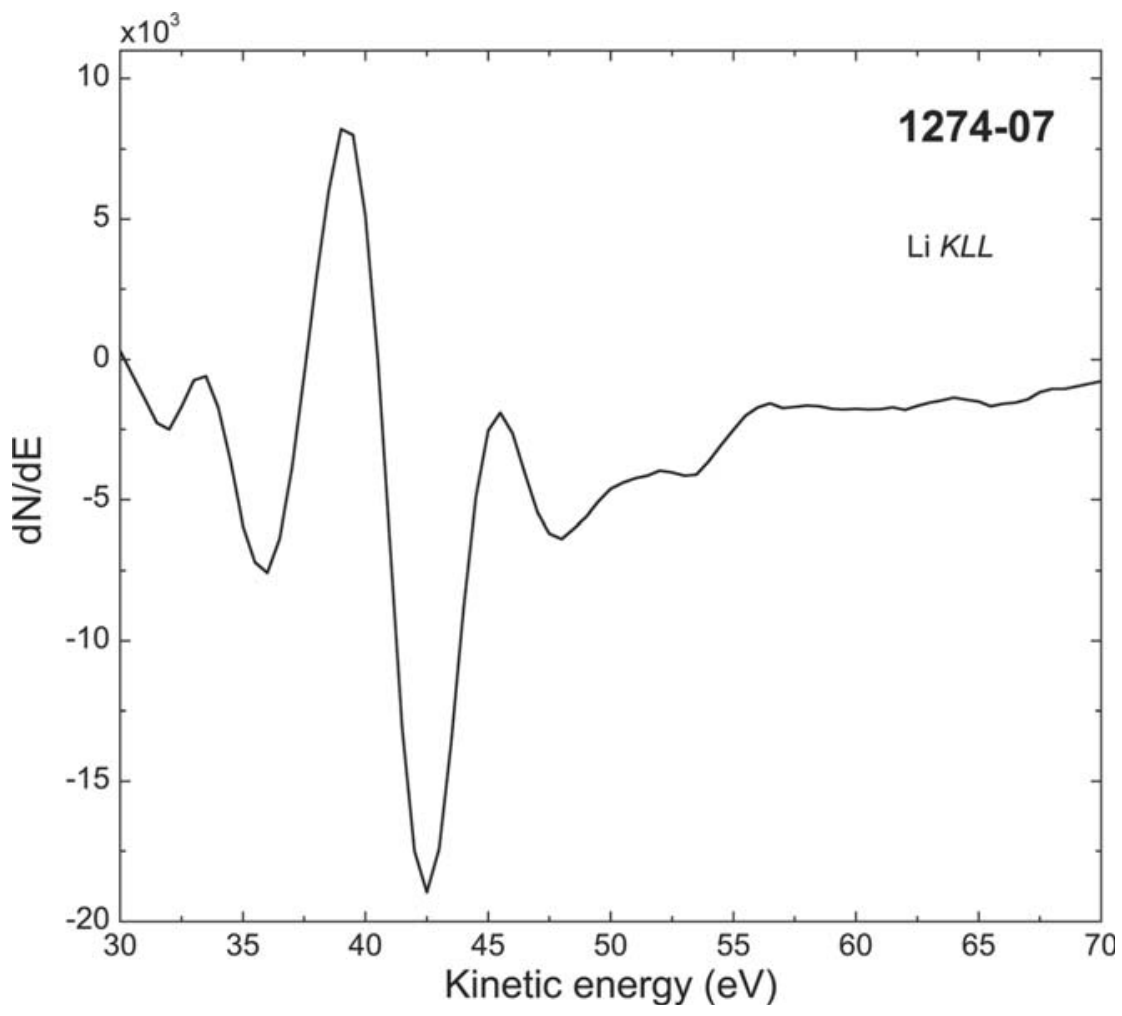

124 Surface Science Spectra, Vol. 20, 2013 


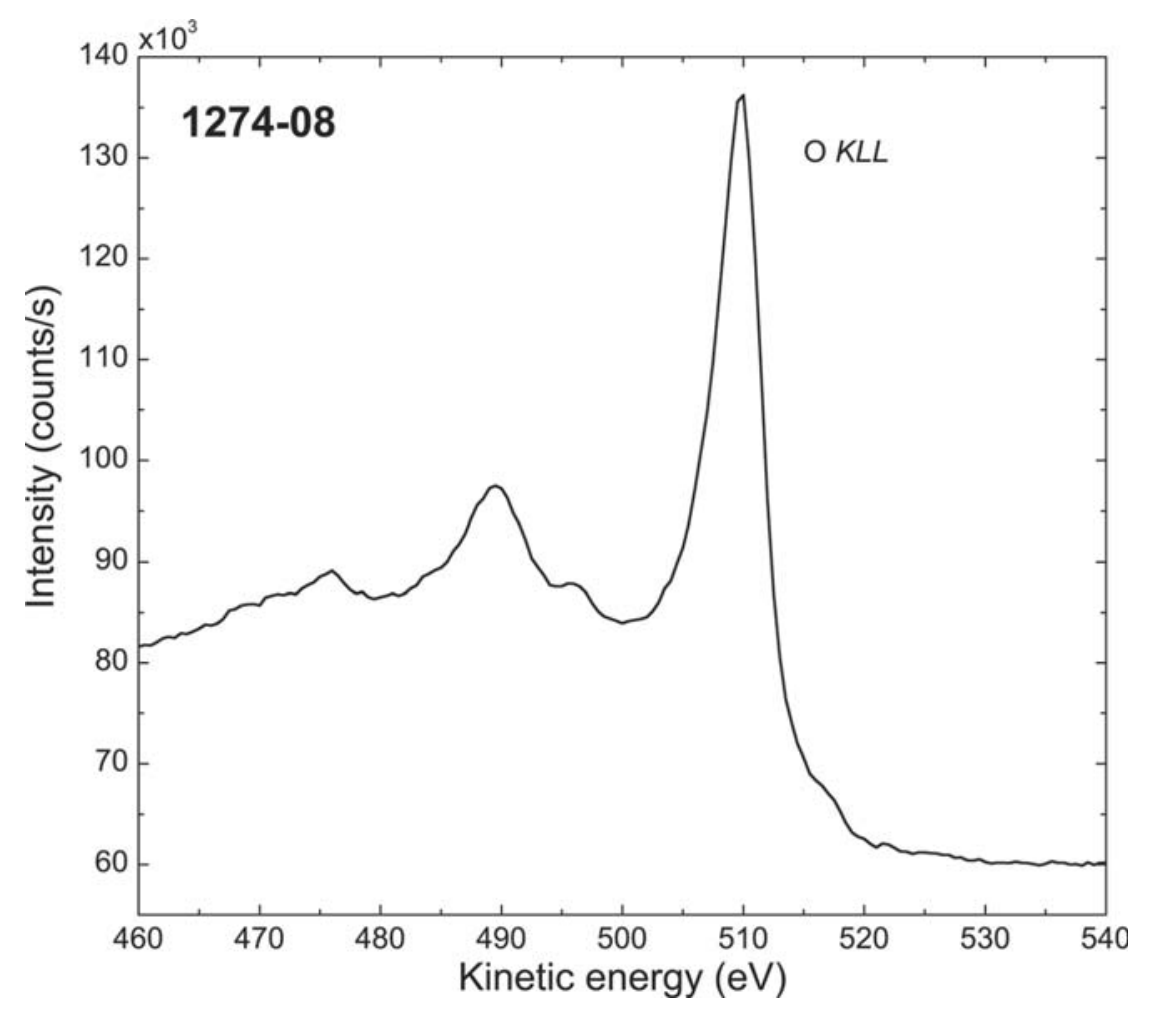

- Accession \#: 01274-08

nost Material: lithium

- Technique: AES

- Spectral Region: O KLL

Instrument: Thermo MicroLab 350

Excitation Source: electron beam

Source Energy: $10000 \mathrm{eV}$

Source Strength: $1.2 \mathrm{nA}$

Source Size: $0.1 \mu \mathrm{m} \times 0.1 \mu \mathrm{m}$

Analyzer Type: spherical sector

Incident Angle: $30^{\circ}$

Emission Angle: $75^{\circ}$

Analyzer Retard Ratio: 4

Analyzer Resolution: 2\%

Total Signal Accumulation Time: $402 \mathrm{~s}$

Total Elapsed Time: $510 \mathrm{~s}$

Number of Scans: 50

Source Beam Size at Specimen Surface: $0.011 \mu \mathrm{m} \times 0.011 \mu \mathrm{m}$

Effective Detector Width: $0.5 \mathrm{eV}$

Analyzer Angular Acceptance Width: $25^{\circ} \times 25^{\circ}$

Comment: oxide particle embedded in Li sample, take-off angle $=15^{\circ}$ 


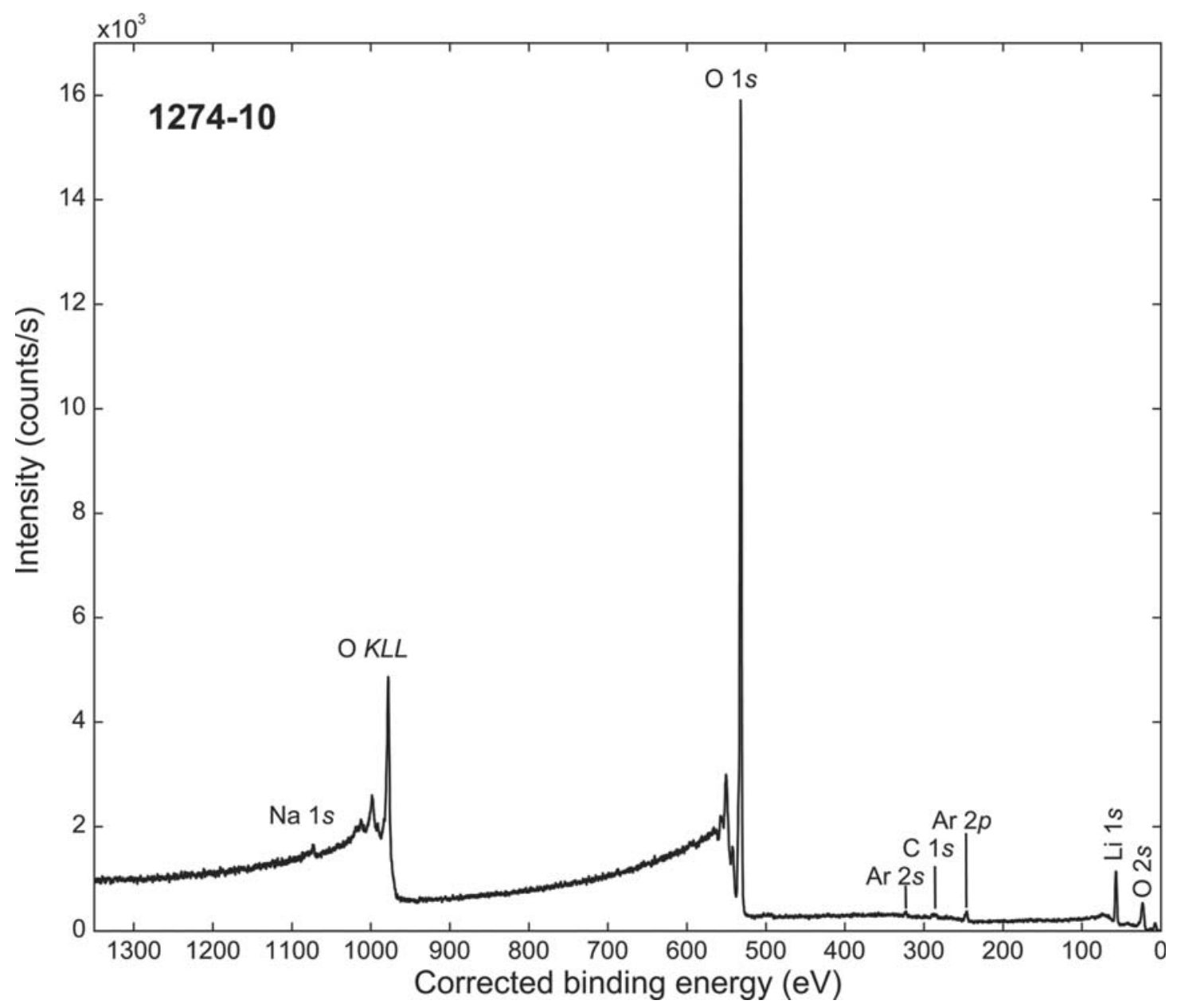

\begin{tabular}{rc}
\hline Accession \# & $01274-10$ \\
Host Material & lithium \\
Technique & XPS \\
Spectral Region & survey \\
Instrument & Thermo Scientific Theta Probe \\
Excitation Source & Al $K_{\alpha}$ monochromatic \\
Source Energy & $1486.68 \mathrm{eV}$ \\
Source Strength & $300 \mathrm{~W}$ \\
Source Size & $200 \mu \mathrm{m} \times 200 \mu \mathrm{m}$ \\
Analyzer Type & spherical sector \\
Incident Angle & $30^{\circ}$ \\
Emission Angle & $53^{\circ}$ \\
Analyzer Pass Energy: & $300 \mathrm{eV}$ \\
Analyzer Resolution & $2.0 \mathrm{eV}$ \\
Total Elapsed Time & $675 \mathrm{~s}$ \\
Number of Scans & $1235 \mathrm{~s}$ \\
Total Signal Accumulation Time & 3 \\
Source Beam Size at Specimen Surface & $400 \mu \mathrm{m} \times 200 \mu \mathrm{m}$ \\
Effective Detector Width & $0.3 \mathrm{eV}$ \\
Analyzer Angular Acceptance Width & $30^{\circ} \times 30^{\circ}$ \\
Comment & surface of Li sample exposed to atmosphere for $<1 \mathrm{~min}$, take-off angle $=30^{\circ}$ \\
\hline
\end{tabular}




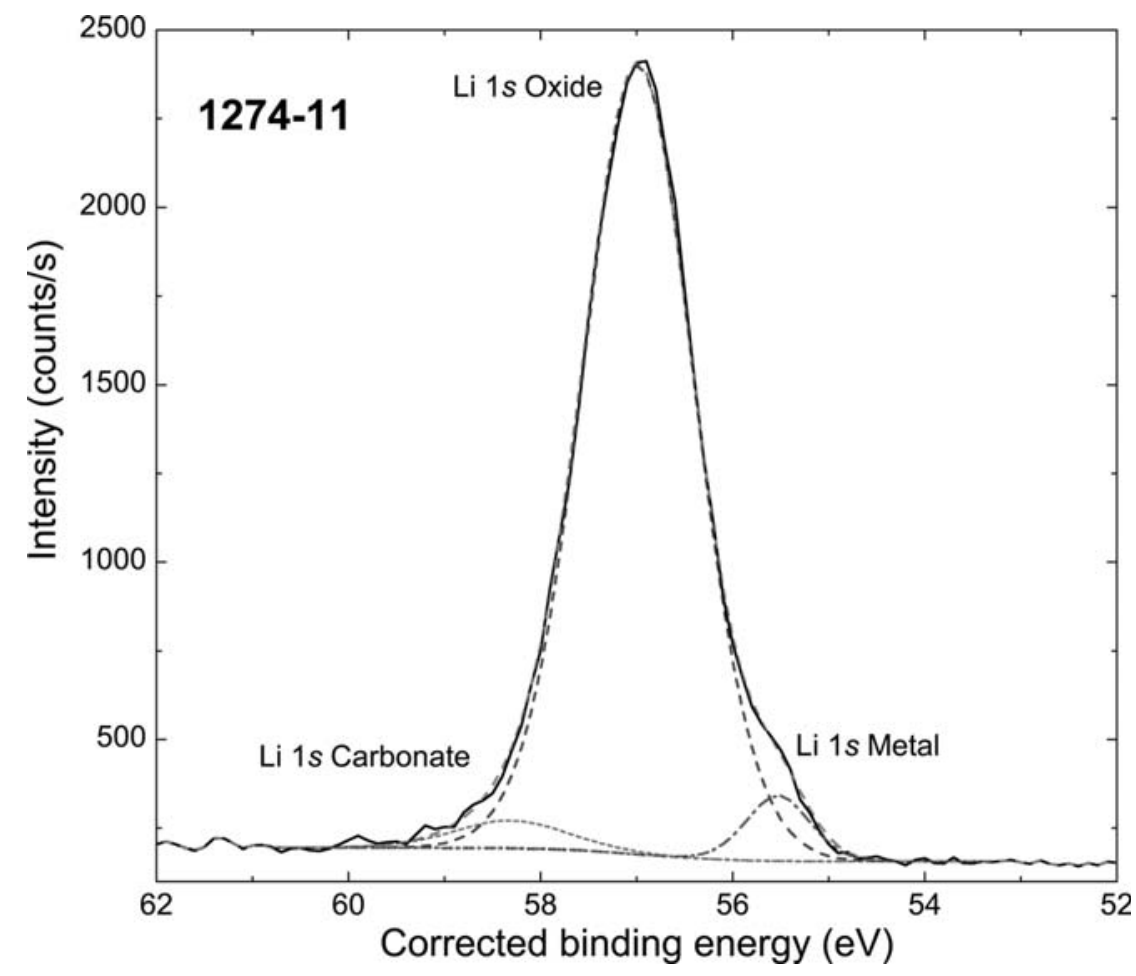

- Accession \#: 01274-11

- Host Material: lithium

- Technique: XPS

- Spectral Region: Li 1s

Instrument: Thermo Scientific Theta Probe

Excitation Source: Al $K_{\alpha}$ monochromatic

Source Energy: $1486.68 \mathrm{eV}$

Source Strength: $300 \mathrm{~W}$

Source Size: $400 \mu \mathrm{m} \times 200 \mu \mathrm{m}$

Analyzer Type: spherical sector

Incident Angle: $30^{\circ}$

Emission Angle: $53^{\circ}$

Analyzer Pass Energy: $80 \mathrm{eV}$

Analyzer Resolution: $0.8 \mathrm{eV}$

Total Signal Accumulation Time: $378 \mathrm{~s}$

Total Elapsed Time: $1020 \mathrm{~s}$

Number of Scans: 50

Source Beam Size at Specimen Surface: $400 \mu \mathrm{m} \times 200 \mu \mathrm{m}$

Effective Detector Width: $0.1 \mathrm{eV}$

Analyzer Angular Acceptance Width: $30^{\circ} \times 30^{\circ}$

Comment: surface of Li sample exposed to atmosphere for $<1 \mathrm{~min}$, take-off angle $=30^{\circ}$

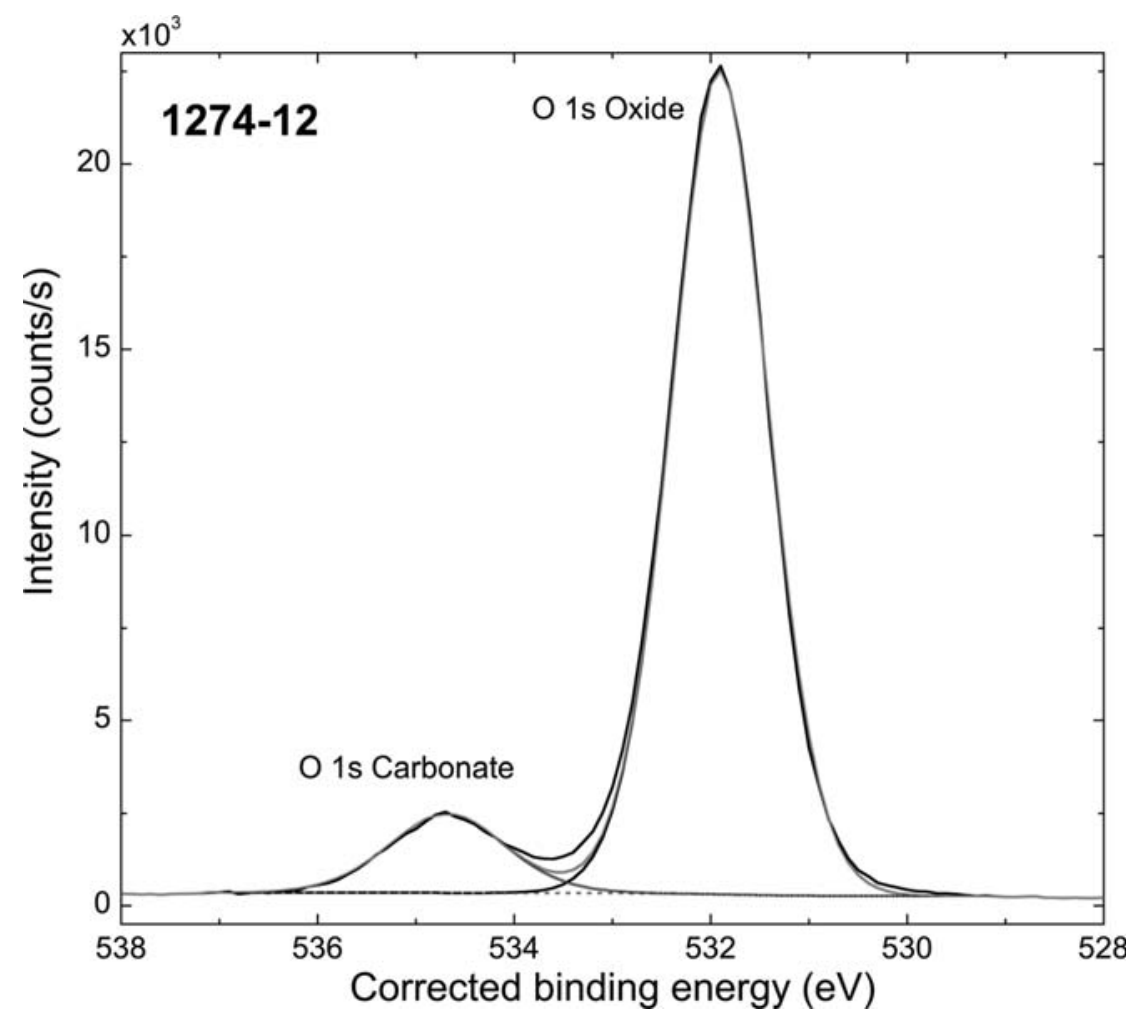

- Accession \#: 01274-12

- Host Material: lithium

- Technique: XPS

- Spectral Region: $01 s$

Instrument: Thermo Scientific Theta Probe

Excitation Source: Al $K_{\alpha}$ monochromatic

Source Energy: $1486.68 \mathrm{eV}$

Source Strength: $300 \mathrm{~W}$

Source Size: $400 \mu \mathrm{m} \times 200 \mu \mathrm{m}$

Analyzer Type: spherical sector

Incident Angle: $30^{\circ}$

Emission Angle: $53^{\circ}$

Analyzer Pass Energy: $50 \mathrm{eV}$

Analyzer Resolution: $0.65 \mathrm{eV}$

Total Signal Accumulation Time: $189 \mathrm{~s}$

Total Elapsed Time: $420 \mathrm{~s}$

Number of Scans: 25

Source Beam Size at Specimen Surface: $400 \mu \mathrm{m} \times 200 \mu \mathrm{m}$

Effective Detector Width: $0.1 \mathrm{eV}$

Analyzer Angular Acceptance Width: $30^{\circ} \times 30^{\circ}$

Comment: surface of Li sample exposed to atmosphere for $<1 \mathrm{~min}$, take-off angle $=30^{\circ}$ 